\title{
El estudio de factibilidad en la gestión de los proyectos de inversiones
}

\author{
Roberto Delgado Victore ${ }^{1}$ \\ María Antonia Vérez García ${ }^{2}$
}

Recibido: 1 de mayo de 2015

Aprobado: 15 de mayo de 2015

\author{
Delgado, R. y Vérez, M. (2015). El estudio de factibilidad en la \\ gestión de los proyectos de inversiones. Activos, 24, 177-196. \\ Clasificación JEL \\ G-11
}

\section{Resumen}

El trabajo tiene como objetivo brindar un procedimiento para el desarrollo del estudio de factibilidad a lo largo del ciclo de vida del proyecto de inversión en el sistema del turismo en Cuba. Se apoya en el uso de los indicadores para el desarrollo de su estrategia de dirección.

En el desarrollo del tema se muestran las funciones del estudio de factibilidad en el subproceso de preinversión como medio de evaluación de los anteproyectos en la ingeniería básica, su uso en la ingeniería de detalle, la ejecución y el control, para finalmente validar al cierre del proyecto y el cumplimiento de los indicadores previamente definidos en el estudio de factibilidad.

1 Profesor titular. ISPJAE. Cuba. Correo electrónico: robertodv@uci.cu

2 Profesora auxiliar. Facultad de Contabilidad y Finanzas. Universidad de La Habana. Cuba. Correo electrónico: mara@fcf.uh.cu 


\title{
Palabras claves
}

Estudio de factibilidad, proyecto, inversiones.

Delgado, R. y Vérez, M. (2015). The feasibility study on the management of investment projects. Activos, 24, 177-196.

\begin{abstract}
The work has as objective, to offer a procedure for the development feasibility study along the cycle life project investment in the tourism system, supported by the use development indicators of its strategy management.

In the topic development, the functions of the feasibility study are shown in the initial investment process, like half preliminary evaluation in the basic engineering designs, their use in the detail engineering, the execution and control, for finally, to validate the project closing, the indicators execution, previously defined in the feasibility study.
\end{abstract}

\section{Keywords}

Feasibility study, project management.

Delgado, R. y Vérez, M. (2015). L'étude de faisabilité dans la gestion des projets d'investissements. Activos, 24, 177-196.

\section{Résumé}

Le travail a pour objectif $\mathrm{d}$ 'apporter une procédure pour le développement de l'étude de faisabilité tout au long du cycle de vie du projet $\mathrm{d}$ 'investissement dans le système du tourisme à Cuba. Il s'appui sur l'utilisation des indicateurs pour le développement de sa stratégie de direction. Dans le développement du thème, on montre les fonctions de 
l'étude de faisabilité dans le sub-processus de pré-investissement comme méthode d'évaluation des avant-projets dans l'ingénierie basique, son utilisation dans l'ingénierie de détail, l'exécution et le contrôle, pour finalement valider à la fermeture du projet et l'application des indicateurs définis précédemment dans l'étude de faisabilité.

\section{Mots clés}

Étude de faisabilité, projet, investissements.

\section{Introducción}

El sistema de dirección y gestión de los proyectos del turismo en Cuba necesita de un sistema de evaluación de proyectos que le permita, por medio de un conjunto de indicadores, establecer las prioridades de la demanda de proyectos en función de la disponibilidad real que brindan las fuentes de financiamiento tanto nacionales como internacionales.

El estudio de factibilidad es la base para la toma de decisiones de los directivos que tienen la responsabilidad de aprobar las inversiones, para lo cual atienden a los valores de los indicadores, a saber, periodo de recuperación (PR), valor actual neto (VAN) y tasa interna de retorno (TIR), para establecer la estrategia de ejecución de las inversiones en función de las prioridades según los indicadores y las fuentes de financiamiento disponibles. La acertada toma de decisiones de los directivos depende de la calidad de la fuente de información, su organización, tratamiento y proceso de cálculo, en el que el MS Project 2013 y el Excel, como herramientas informáticas, tienen una buena aplicación en el proceso de dirección del turismo.

La estrategia del uso del financiamiento disponible debe estar basada en un estudio que permita optimizar el uso de los recursos y ponerlos donde mayor rendimiento económico, técnico, financiero y social brinden al desarrollo del país. Este objetivo es posible cuando la estrategia financiera 
se fija a partir de un sistema efectivo, en el que el estudio de factibilidad ofrezca la información necesaria mediante los indicadores, para determinar las prioridades y desarrollarlas en función del financiamiento disponible.

El objetivo del trabajo es brindar un procedimiento para el desarrollo del estudio de factibilidad de los proyectos en el sistema de dirección y gestión en el sector del turismo, con el propósito de implantar una secuencia de ejecución de los proyectos de inversiones, atendiendo a las prioridades en función de los indicadores que los estudios aportan.

En el desarrollo del tema se expresan los conceptos fundamentales, las fases de la gestión del proyecto en su ciclo de vida, las funciones del estudio de factibilidad en cada una de estas fases a partir de lo establecido en el Decreto Ley n. ${ }^{\circ} 327$ del Consejo de Ministros, "Reglamento sobre las inversiones", las normas ISO asociadas al desarrollo de los proyectos y el Project Management Institute (ISO, 2012) de 2012 (PMI), según el Project Management Body of Knowledge (PMBOK), haciendo uso de herramientas como MS Project 2013 y los complementos del Office.

\section{Desarrollo}

El ciclo de vida de la inversión se inicia con el proceso de preinversión, en el que se desarrollan los anteproyectos, siendo el estudio de factibilidad el que permitirá definir cuál de ellos es viable y entonces proceder a determinar el presupuesto. Durante su ciclo de vida se desarrollan los estudios de riesgos, las defectaciones, los avales necesarios, el estudio de factibilidad, el presupuesto directivo y el contrato, todo en el marco del proyecto técnico. En el proceso se desarrolla el flujo de caja, se comprueba su sustentabilidad y la sostenibilidad según el esquema de su variación, en función del número de años de diseño de la inversión. 


\section{El estudio de factibilidad como estrategia de ejecución de proyectos}

El problema de tener más proyectos por ejecutar que disponibilidad financiera se presenta con frecuencia y es muy importante. En estos casos se hace imprescindible el desarrollo de los estudios de factibilidad para determinar las prioridades, identificar los indicadores y, a partir de su ponderación, establecer un orden de prioridades para definir la estrategia de ejecución.

En el análisis de las prioridades de los proyectos para determinar las estrategias de ejecución en función de la disponibilidad de financiamiento se puede presentar un conjunto de variantes en las cuales se analizan los valores relativos de los indicadores que son claves, como el PR, el VAN y la TIR; el VAN social y el costo/beneficio pueden ser usados como complementos en algunos casos. El uso de una matriz con los indicadores del estudio de factibilidad, y otros que se consideren de interés, permite establecer las prioridades para la estrategia de ejecución de los proyectos, dejando el resto en cartera. El entorno en el tiempo de evaluación de los indicadores debe ser común para el conjunto de proyectos (De La Oliva, 2001).

\section{El estudio de factibilidad en el subproceso de preinversión}

El subproceso de preinversión es el subproceso conceptual directivo en el que se define qué es necesario hacer para resolver una necesidad propuesta por un cliente. Por medio de los requerimientos se desarrolla un programa de trabajo en el cual se planifican las acciones para desarrollar su ejecución y el fin del proceso. Este proceso se conoce como concepción, ingeniería básica o iniciación, y es en él donde se desarrolla el proyecto técnico; se apoya en la metodología de la investigación, la ciencia del proyecto, el PMBOK, las normas ISO, el método científico y el marco lógico. El proceso de licitación se estimula como forma de elevar la calidad y la competitividad. 
En el subproceso de preinversión se desarrollan un conjunto de acciones que conducen a un proceso cíclico y repetitivo en función de garantizar su calidad. La figura 1 muestra las acciones relacionadas con el estudio de factibilidad, donde los anteproyectos generan la documentación principal para el estudio, con los indicadores del periodo de recuperación, el valor actual neto (VAN), la tasa interna de retorno (TIR) y el costo/beneficio como medios de evaluación para la correspondiente toma de decisiones. Un VAN positivo indica que los flujos futuros que se espera que la inversión genere serán mayores que el costo en el que hay que incurrir para su ejecución. Una TIR por encima de la tasa de descuento o costo de oportunidad indica que el rendimiento esperado de la inversión es mayor que el costo en el que hay que incurrir para su financiamiento.

Figura 1. Modelo para la preinversión del proyecto

\section{Inicio Propuesta de proyecto del cliente Acta de constitución \\ Proceso de concepción}

Selección del director de proyecto

Organización Equipo.

Contenido del proceso

Tarea técnica

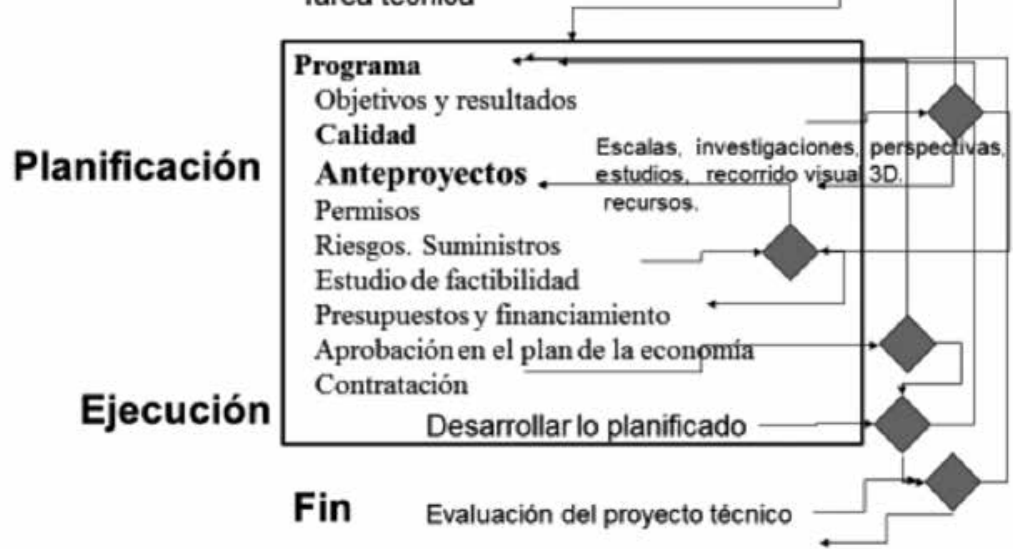

Fuente: elaboración propia. 
Los indicadores reflejan el comportamiento estratégico de la inversión. De la calidad de la información y de su proceso de cálculo dependen las decisiones que se tomen.

En el subproceso de preinversión se preparan las condiciones para el proceso de diseño, planificación, ingeniería de detalle o formulación del proyecto, subproceso en el que el estado del arte, el comportamiento de los proyectos ejecutados por la empresa y reflejados en una base de datos de proyectos terminados, las proyecciones estratégicas de la empresa con los escenarios donde se obtendrán los resultados, así como los criterios de medidas de los objetivos reflejados en los resultados previstos y el control sistemático de los indicadores de costo, tiempo, calidad, logística y desempeño -mediante la aplicación del método científico apoyado por las tecnologías de la información y las comunicaciones (TIC) - garantizan el éxito del nuevo proyecto. El proceso estimula y desarrolla la creatividad y la innovación del hombre que ejecuta las acciones. El proceso de preinversión se apoya en la base de datos de proyectos terminados, con el objetivo de utilizar los módulos y componentes ya elaborados en proyectos anteriores con el uso de las plantillas, las bibliotecas, los indicadores globales y las experiencias desarrolladas en los procedimientos ejecutados, con el propósito de garantizar la calidad de la documentación en un proceso de mejora continua que garantiza su perfeccionamiento.

El proceso contiene las ideas conceptuales, define lo que es necesario hacer y, dependiendo del alcance del proyecto, puede contener el estudio de oportunidad y prefactibilidad. En ocasiones, en una instalación es necesario evaluar previamente, por ejemplo, si esta se demuele o se conserva, para continuar con el proceso de preinversión.

\section{El estudio de sensibilidad}

Todos los proyectos de inversión están sujetos a numerosos riesgos e incertidumbre, tanto por la calidad de la información como por los procesos de cálculo utilizados. El proceso de estimación de los valores que reflejan el 
comportamiento de los proyectos ejecutados en la base de datos de proyectos terminados es otro aspecto importante que se debe tener presente en el estudio de los indicadores clave, como el VAN, el PR y la TIR.

El estudio del entorno lógico de las variables clave que inciden en los indicadores posibilita conocer el rango de posibles cambios por realizar en el ciclo de vida del proyecto, sin afectar la funcionalidad y el alcance aprobado en el estudio de factibilidad. También permite a los proyectistas conocer el grado de libertad disponible en la ingeniería de detalle, para realizar cambios o ajustes dentro del marco de las cifras directivas dadas en la ingeniería básica, supervisado por el inversionista. A su vez, hace posible la evaluación del rango de variación en las órdenes de cambio, los suplementos al contrato y los incrementos de financiamiento. Este tipo de estudio es parte del instrumento de trabajo de que dispone el inversionista para gestionar los cambios durante el ciclo de vida del proyecto, con la finalidad de garantizar la sostenibilidad en el ciclo de vida de la inversión. Además, es una forma de evaluar los posibles riesgos que el proyecto de inversión pueda tener.

El estudio de las posibles variaciones de los indicadores en el estudio de factibilidad se realiza mediante el estudio de sensibilidad, aunque también puede usarse el estudio de escenarios y el método de simulación Monte Carlo. A pesar de su desventaja de mover la variable de mayor incertidumbre manteniendo el resto constante, el análisis de escenarios es más utilizado y más avanzado, pues tiene en cuenta la combinación de varias variables y la asignación de probabilidades a los escenarios. Por su parte, el método de la simulación Monte Carlo requiere nivel de información para su cálculo.

Las conclusiones que resulten del análisis de sensibilidad pueden conducir al replanteamiento del proyecto como consecuencia de la inseguridad en los resultados obtenidos, y en situaciones extremas, llegar incluso a desistir en su ejecución. Este estudio permite evaluar de mejor forma las oportunidades y las amenazas, para aprovechar las fortalezas y combatir las posibles debilidades durante su ciclo de vida. 


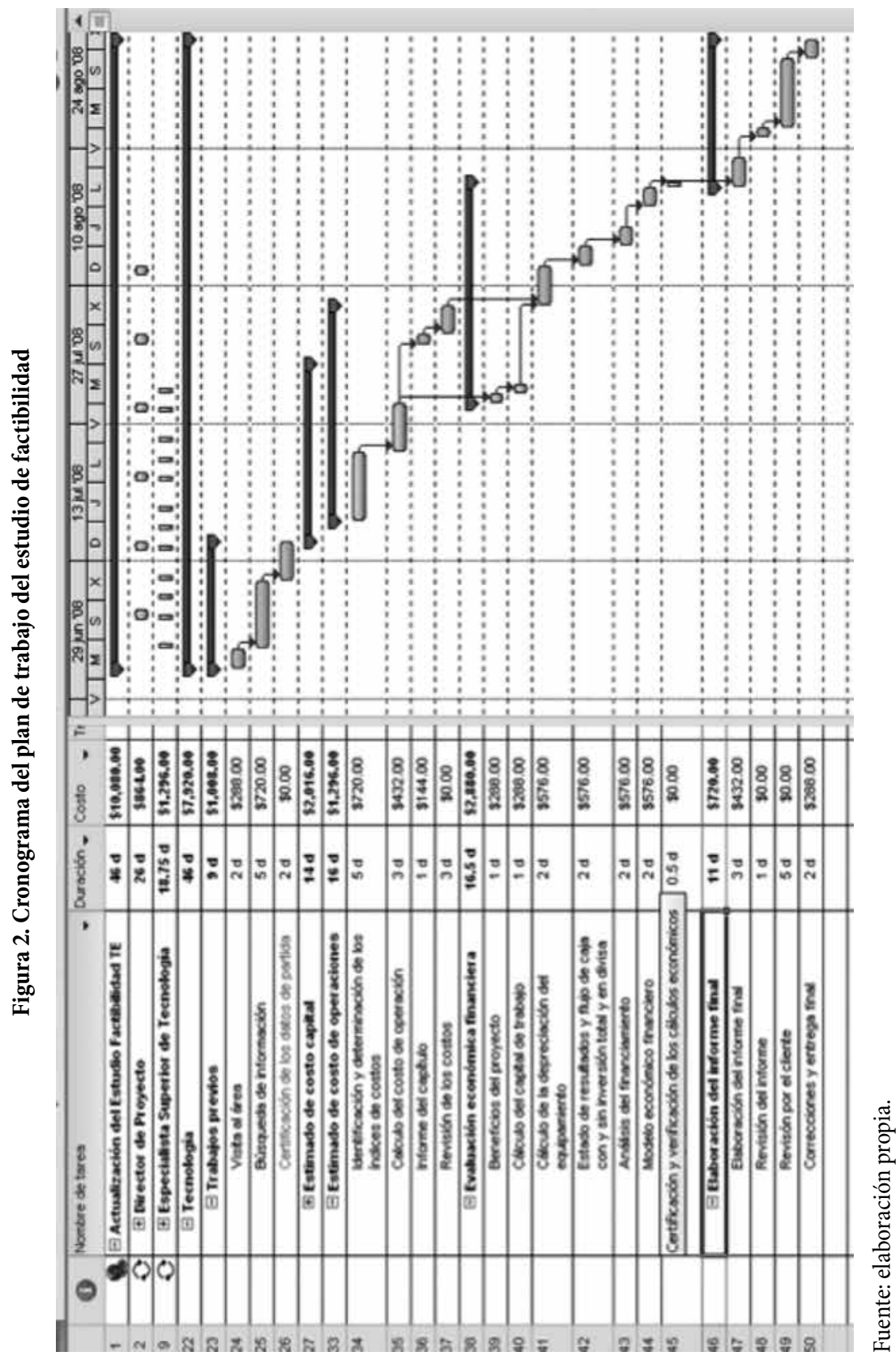


A modo de ejemplo, en la figura 2 se muestra el cronograma de ejecución del estudio de factibilidad representado como subproyecto del proyecto de inversiones en la fase de la preinversión, y forma parte del cronograma general para el proceso de preinversión del proyecto. El uso del MS Project 2013 para el diseño y control del proyecto de inversiones permite evaluar y controlar por cortes el desarrollo integral del proyecto de inversiones.

\section{El flujo de caja del estudio de factibilidad apoyado por el Excel}

En el estudio de factibilidad para una inversión Ao, el flujo de caja está representado por un conjunto de entradas y salidas, con sus diferencias a favor de las entradas por años, que van reduciendo el valor de Ao hasta alcanzar el periodo de recuperación, que es posible obtener a partir del uso de la herramienta informática que brinda Excel.

En la figura 3 (Flujo de caja estructurado por hojas en el Excel) se muestra el contenido de un libro integrado por un conjunto de hojas con los consolidados que alimentan la hoja principal del flujo de caja según su estructura. La hoja principal muestra las entradas y salidas con la desagregación de sus partes componentes, en función del tipo de proyecto y las acciones por evaluar. Las distintas partes componentes de las entradas y salidas se desarrollan en hojas independientes, en las cuales se realiza el desglose con las integraciones por años para alimentar la hoja principal del flujo de caja.

Para el desarrollo de las siguientes hojas de cálculo, como los gastos de operaciones, el capital de trabajo y demás funciones, el desarrollo es similar, como se muestra en la figura 3. El cálculo del VAN, el PR y la TIR se calculan de la siguiente forma: 
El estudio de factibilidad en la gestión de los proyectos de inversiones $\mid$ ACTIVOS 187

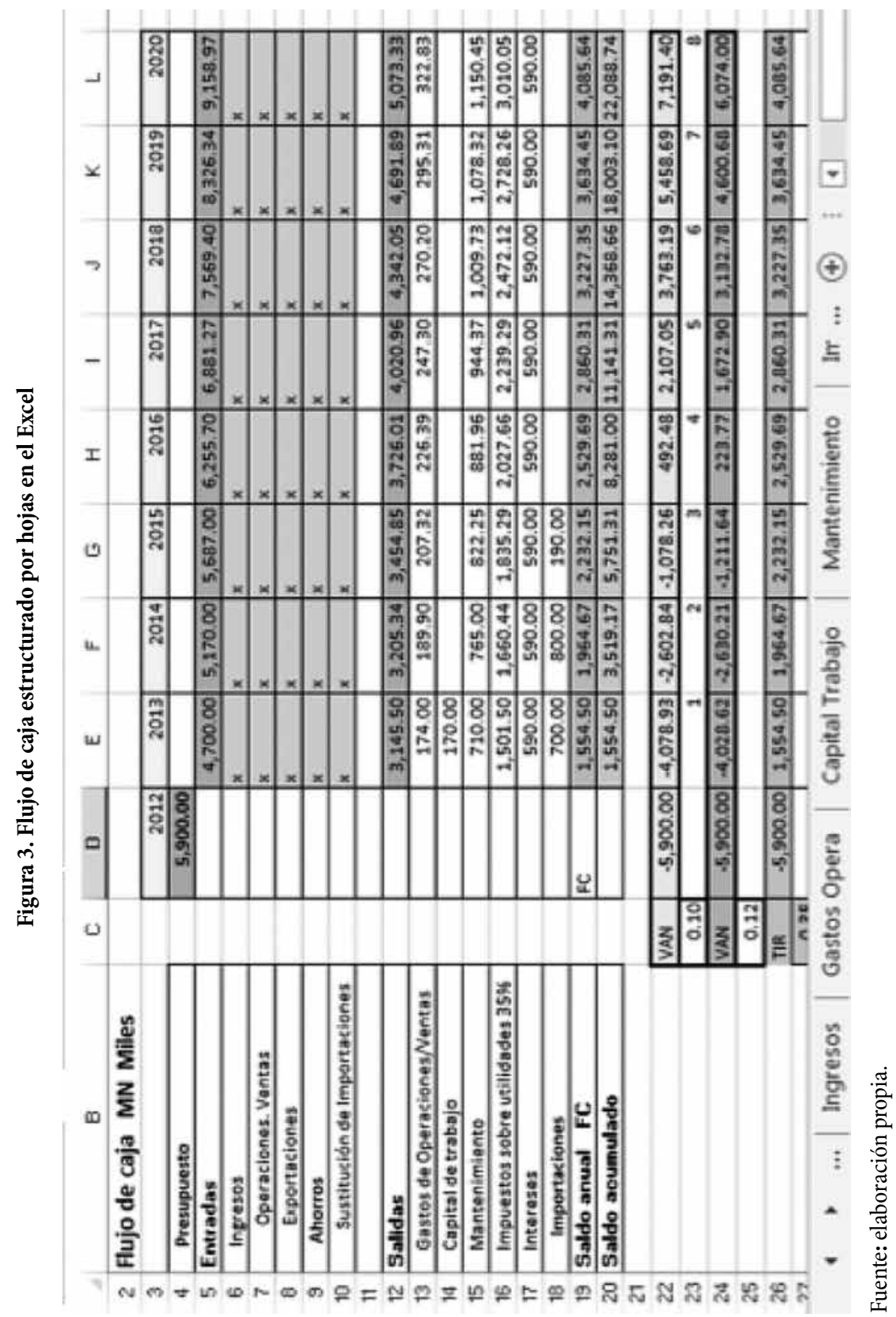


Figura 4. Distribución de los ingresos por partidas

\begin{tabular}{|c|c|c|c|}
\hline \multicolumn{4}{|l|}{ Datos de la inversión } \\
\hline & Total & CuC & CUP \\
\hline Presupuesto inversión & 5900.0 & & \\
\hline Construcción y montaje & 2800 & 1050 & 1750 \\
\hline Demolición & 1500 & 500 & 1000.0 \\
\hline Edificación & 1200 & 500 & 700.0 \\
\hline Suministros & 100 & 50 & 50.0 \\
\hline Equipos & 1900 & 900 & 1000 \\
\hline Clima & 1400 & 700 & 700.0 \\
\hline Elevador & 500 & 200 & 300.0 \\
\hline Otros & 1200.0 & 500.0 & 700.0 \\
\hline Estudio de factibilidad & 800.0 & 400 & 400.0 \\
\hline Capital de trabajo & 400.0 & 100 & 300.0 \\
\hline Servicios bancarios & 300.0 & 100 & 200.0 \\
\hline
\end{tabular}

Fuente: elaboración propia.

Figura 5. Distribución de las partidas de mantenimiento

\begin{tabular}{|c|r|r|r|r|r|r|r|r|r}
\hline & 2012 & 2013 & 2014 & 2015 & 2016 & 2017 & 2018 & 2019 & 2020 \\
\hline Mantenimiento & & 710 & 765 & 822.25 & 881.96 & 944.37 & 1009.7 & 1078.3 & 1150.5 \\
\hline Interno & & 410 & 440 & 470 & 500 & 530 & 560 & 590 & 620 \\
\hline Salario & & 60 & 60 & 60 & 60 & 60 & 60 & 60 & 60 \\
\hline Medios & & 150 & 170 & 190 & 210 & 230 & 250 & 270 & 290 \\
\hline Piezas & & 200 & 210 & 220 & 230 & 240 & 250 & 260 & 270 \\
\hline Estructura & & & & & & & & & \\
\hline & & & & & & & & & \\
\hline Externo & & 300 & 325 & 352.25 & 381.96 & 414.37 & 449.73 & 488.32 & 530.45 \\
\hline Servicios & & 200 & 220 & 242 & 266.2 & 292.82 & 322.1 & 354.31 & 389.74 \\
\hline Equipamiento & & 100 & 105 & 110.25 & 115.76 & 121.55 & 127.63 & 134.01 & 140.71 \\
\hline & & & & & & & & & \\
\hline
\end{tabular}

Fuente: elaboración propia.

\section{VAN: Valor actual neto}

Es el valor actualizado del flujo de ingresos netos obtenidos durante el ciclo de vida de la inversión, a partir de la determinación por año de las entradas y salidas de efectivo por medio del flujo de caja, desde que se incurre en el 
primer gasto de inversión durante el proceso de preinversión del proyecto hasta que los años de operación o funcionamiento de la inversión concluyen. Un VAN positivo indica que los flujos futuros que se espera genere la inversión serán mayores que el costo en que hay que incurrir para su ejecución (Castro Tato, 2001).

La figura 3 ilustra un ejemplo en el que se muestra el cálculo del VAN para una tasa de interés $(r)$ de $r=0.10$. Aplicando su función $=V N A(\$ C 23$, $\$ D 22, \mathrm{E} 19)=(0.10,-\$ 5900, \$ 1554.50)=-\$ 4078.93$ para $2013 \mathrm{y}$ desplazando la función hasta 2020, se obtienen los valores del VAN por años, que son representados gráficamente en la hoja del Excel.

\section{PR: Periodo de recuperación}

Para ejemplificar el cálculo del periodo de recuperación, en la figura 6 se muestra la forma de obtener el gráfico a partir del flujo de caja mostrado en la figura 3, aplicando la fórmula del cálculo del VAN $=\mathrm{f}(\mathrm{FC}, \mathrm{n})=0$ para $\mathrm{r}=0.1$, donde $\mathrm{n}=3.8$ años.

Figura 6. Periodo de recuperación

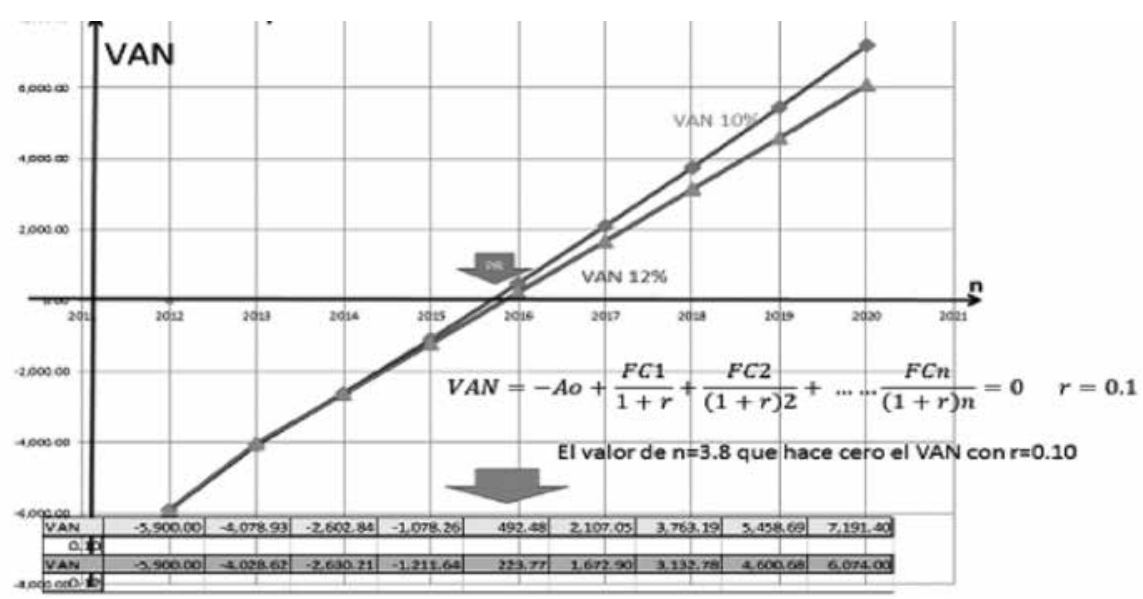

Fuente: elaboración propia. 


\section{TIR: Tasa interna de retorno}

La TIR de una inversión es el valor del interés $r$ que anula el VAN para un valor de n que define el flujo de caja. El VAN está relacionado con el flujo de caja, la tasa de interés y la TIR. Para un valor de n, una vez definida la serie del flujo de caja, se define el VAN, y el valor de r que hace cero el VAN es la TIR. VAN $(\operatorname{TIR}(. .),. \ldots)=0$. Una TIR por encima de la tasa de descuento 0 costo de oportunidad indica que el rendimiento esperado de la inversión es mayor que el costo en que hay que incurrir para su financiamiento.

$$
\mathrm{VAN}=-\mathrm{Ao}+\mathrm{r}=0.10
$$

En el ejemplo del flujo de caja se obtiene el gráfico del VAN $=\mathrm{f}(\mathrm{FC}$, n) para $\mathrm{r}=0.1$, donde el valor del VAN para $\mathrm{n}=8$ es $\mathrm{VAN}=\$ 7191.40$ con $\mathrm{r}=0.1$. Tomando este como punto de partida para la curva VAN $=\mathrm{f}(\mathrm{r})$ para $\mathrm{n}=8$, se obtienen los distintos valores del VAN haciendo variar $\mathrm{r}$, donde, mediante interpolación, se determina el valor de la TIR $=0.35$ en el momento en que el VAN $=0$. Este es el procedimiento interpolado aplicando el concepto de obtención de la TIR donde el VAN es cero. De forma directa en el Excel es posible obtener la TIR aplicando la función como se muestra en la figura 7 por $\mathrm{fx}=\mathrm{TIR}$ (D26:L26, estimado). De manera que $\mathrm{fx}=\mathrm{TIR}$ (\$ 5900: \$ $4085.64,0.4$ ).

Figura 7. Cálculo de la TIR en función del VAN y r
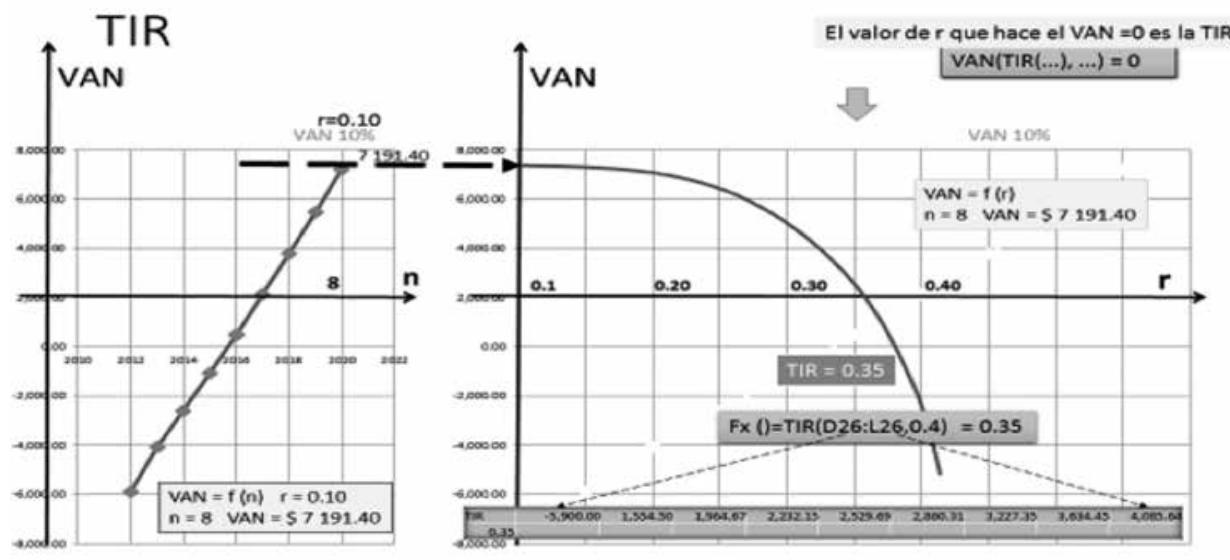

Fuente: elaboración propia. 


\section{El estudio de factibilidad en el subproceso de formulación}

Esta fase de la ingeniería de detalle se desarrolla teniendo presentes todas las definiciones dadas en el proceso anterior de ingeniería básica, en el que se definen las cifras directivas y los indicadores del estudio de factibilidad.

En factibilidad se desarrolló el estudio de sensibilidad en el que se evaluaron los rangos posibles de variación de los parámetros claves. En esta fase, el proyectista desarrolla la ingeniería de detalle sobre la base de las cifras directivas, la funcionalidad y el alcance definido en el estudio de factibilidad, con las posibles variaciones de rango que el análisis de sensibilidad le brinda. Las variaciones que requieran ajustes en el alcance definido en el estudio de factibilidad tienen que ser aprobadas por el nivel que aprobó el estudio de factibilidad en la ingeniera básica. Este concepto limita las posibilidades liberales de los cambios, que generan ajustes con posibles riesgos, ajustes de contratos y suplementos, los cuales producen incrementos de costos en el presupuesto.

\section{El estudio de factibilidad en el subproceso de ejecución}

En el ciclo de vida del proyecto, el proceso de ejecución constituye uno de los más importantes por su complejidad y por la importancia en la toma de las decisiones necesarias para lograr sus objetivos y alcanzar los resultados planificados, acortando el plazo de tiempo en el marco del presupuesto, con el suministro programado, el desempeño del hombre que ejecuta las acciones y con la calidad requerida por el cliente y las partes interesadas. Es la fase en la que se presenta el mayor número de cambios que ajustan el alcance dentro del marco permisible dado por el estudio de factibilidad aprobado por la instancia correspondiente. En este proceso, el ejecutor, apoyado por el inversionista, se opone a los cambios destinados, orientados por fuentes no autorizadas para generar cambios inapropiados en el proyecto en ejecución. El control por cortes para evaluar los cambios, las 
desviaciones, los indicadores del proyecto y los contratos, junto con el análisis de las causas para el desarrollo de la toma de decisiones apoyado por el MS Project 2013, brindan una herramienta importante para la dirección efectiva del proceso inversionista en el turismo.

\section{El estudio de factibilidad en el subproceso de cierre}

En el proceso de cierre del proyecto, se comprueban el cumplimiento de los objetivos, la obtención de los resultados, el costo y su relación con el presupuesto planificado, y el plazo con la calidad requerida por el cliente y las partes interesadas. En el proceso de preinversión se elabora el estudio de factibilidad con su flujo de caja, que es necesario validar en el cierre con el fin de desarrollar un pronóstico de su sostenibilidad durante el ciclo de vida de la inversión (Delgado Victore, 2010).

Este pronóstico puede variar en función del nivel de cumplimiento de la ejecución del proyecto, atendiendo a su calidad, y con el consecuente ajuste del periodo de recuperación, el VAN y la TIR.

En este proceso se evalúa la sustentabilidad y se estudia la sostenibilidad en la puesta en explotación del proyecto a partir de los valores estimados en la preinversión, precisando y ajustando las posibles entradas y salidas para determinar el flujo de caja por intervalos anuales de tiempo. En este momento, el programa de mantenimiento y sus costos tienen una vigencia importante en función de la variación y la tendencia del indicador de la calidad en los cortes del proyecto; si la calidad fue buena, no hay preocupaciones relacionadas con el costo de los mantenimientos en el periodo de explotación; si el indicador refleja valores preocupantes, el costo generalmente se eleva y afecta tanto el periodo de recuperación de la inversión como el valor estimado para la TIR y el VAN. El balance financiero en los cortes -en el que se evalúan la calidad, las no conformidades y el retenido para el financiamiento del periodo de garantía- es un momento en el proceso de dirección en el cual el inversionista toma vivencia de los problemas por resolver durante el ciclo de vida de la inversión y su sostenibilidad. 
En este proceso de cierre del proyecto de inversión se evalúan:

- la evaluación del cumplimiento de lo aprobado como factible,

- la mejora continua en el perfeccionamiento de los estudios de factibilidad,

- la actualización de la base de datos de los proyectos de inversión terminados,

- el ciclo de vida del proyecto y el ciclo de vida de la inversión, y

- la calidad del proyecto y su influencia en el mantenimiento.

Con este análisis, el director de proyecto y el explotador pueden analizar la estrategia del comportamiento de la inversión y en cuál de los escenarios antes proyectados en el análisis de riesgos y sensibilidad se está moviendo la inversión; a partir de ello ambos pueden tomar las medidas pertinentes. Con este estudio se analizan las posibles desviaciones con respecto a lo proyectado, los excesos y los efectos producidos, para realizar luego una nueva proyección con los nuevos sucesos y evaluar su posible comportamiento y sostenibilidad durante el ciclo de vida de la inversión.

\section{Procedimiento para el cálculo de los indicadores}

El procedimiento parte del concepto del interés compuesto dado por la expresión $\mathrm{An}=\mathrm{Ao}(1+\mathrm{r})^{\mathrm{n}}$, donde Ao $=\mathrm{An} /(1+\mathrm{r})^{\mathrm{n}}$ y el VAN $=-A o+\Sigma$ $\mathrm{FCn} /(1+\mathrm{r})^{\mathrm{n}}$ para $\mathrm{r}=0.10$, la que expresa el valor de la inversión inicial Ao, que se contrarresta con las distintas sumas de las diferencias entre las entradas y salidas por años $\mathrm{n}$, dadas por el FC en un intervalo de tiempo dado. En la figura 3, "Flujo de caja estructurado por hojas en el Excel", se muestra el valor de Ao de la inversión inicial y los valores del FC por años.

El cálculo del VAN $=-A o+\sum \mathrm{FCn} /(1+\mathrm{r})^{\mathrm{n}}$ para $\mathrm{r}=0.10$ se determina para un número de años $\mathrm{n}$, $\mathrm{y}$ donde se cumple VAN $=-A o+\Sigma \mathrm{FCn} /(1+\mathrm{r})^{\mathrm{n}}=0$ para $\mathrm{r}=0.10$ se determina el $\mathrm{PR}$, como se muestra en la figura 6 para $\mathrm{n}=3.8$ años.

El VAN de diseño se determina para el punto donde la curva representativa del ciclo de vida de la inversión inicia su meseta. La cifra estimada para proyectos de construcción puede estar a 2.5 del PR. 
Figura 8. Variación del VAN con $\mathbf{n}$ y con $\mathbf{r}$

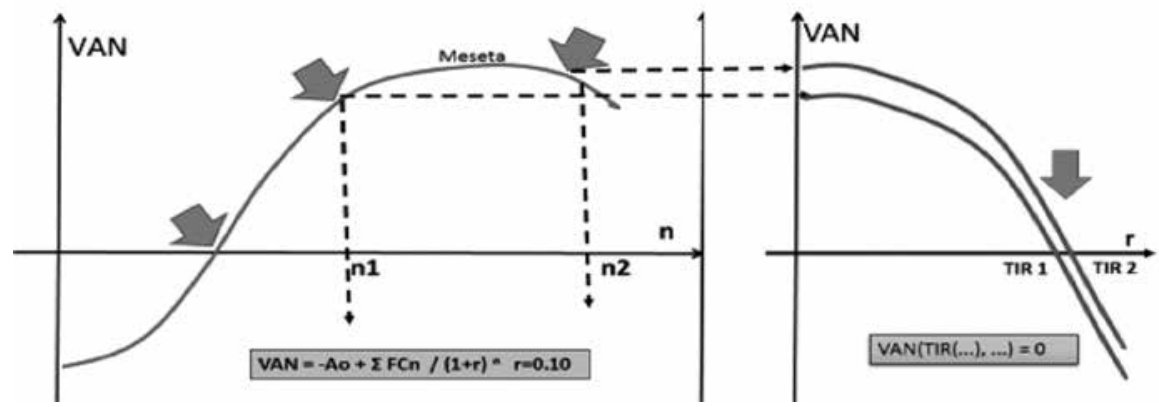

Fuente: elaboración propia.

En la figura 3 "Flujo de caja estructurado por hojas en el Excel", el VAN $=\$ 7191.40$ de diseño se tomó para $\mathrm{n}=8$. La figura 8, "Variación del VAN con n y con r", muestra la variación del VAN $=\mathrm{f}(\mathrm{FC}, \mathrm{n}) \mathrm{r}=0.10$ y el criterio para seleccionar el VAN de diseño en el punto donde se inician la meseta $\mathrm{n}=\mathrm{n} 1$ y la variabilidad de la TIR a partir de este punto. El valor de la TIR se toma para el VAN de diseño interpolando en la curva o con el cálculo estimado, según se muestra en la figura 7 y como se representa en la figura 3 (Delgado Victore, 2010).

\section{Conclusiones}

En el sistema de dirección del turismo, el estudio de factibilidad representa el instrumento que permite a los directivos evaluar las propuestas de proyectos atendiendo a la disponibilidad del financiamiento para aprobar las inversiones y a los valores de los indicadores PR, VAN y TIR, para establecer la estrategia de ejecución de las inversiones en función de las prioridades, y determinar hasta dónde alcanzan las fuentes de financiamiento disponibles. De la calidad de la fuente de información, su organización, tratamiento y proceso de cálculo depende la toma de decisiones de los directivos, en la que, según el Decreto 327, la tecnología del proyecto, el PMBOK y las normas 
ISO, tomando como herramientas el MS Project 2013 y el Excel, tienen una buena aplicación en el sistema de dirección del turismo.

En el contenido se muestra una secuencia lógica del desarrollo del estudio de factibilidad en el contexto de las fases del proceso inversionista dado por el Decreto Ley 327 "Reglamento del Proceso Inversionista".

El estudio de factibilidad permite definir el alcance del proyecto y sus posibles ajustes dentro del entorno definido en el análisis de sensibilidad, lo que representa un instrumento de dirección en el turismo.

Los indicadores del estudio de factibilidad son usados para la toma de decisiones estratégicas de la ejecución de las inversiones y, por tanto, el proceso debe estar sustentado sobre bases de la calidad de la información y el proceso de cálculo de los indicadores.

El estudio de sensibilidad es un importante recurso para el inversionista en la evaluación y aprobación de los posibles cambios dentro del entorno previsto, sin alterar el alcance del proyecto establecido en el estudio de factibilidad.

\section{Referencias bibliográficas}

Acevedo, L. (2010). Evaluación ex post del Proyecto de pan y pastelería congelada y miga para empanar en la empresa Molinera Cárdenas. La Habana, Cuba.

Brealey, R. A., Myers, S. C., \& Giammarino, R. (1992). Principles of Corporate Finance (2. ed.). Canadá: McGraw Hill - Ryerson Limited.

Bolloju, N. (2001). Aggregation of analytic hierarchy process models based on similarities in decision makers' preferences. European Journal of Operational Research, 128(3), 499-508.

Bonilla, R. M. (2011). Gestión de sostenibilidad utilizando lógica borrosa. Madrid: Universidad Complutense de Madrid.

Castro Tato, M. (2001). El valor actual neto (VAN) como criterio fundamental de evaluación de negocios. Revista Economía y Desarrollo, 128(1).

Consejo de Ministros. Decreto Ley 327. Reglamento del Proceso Inversionista. 2014. 
Copeland, T., \& Weston, J. F. (1988). Financial Theory and Corporate Policy (3. ${ }^{\mathrm{a}}$ ed.). U. S. A.: Addison - Wesley Publishing Company.

De La Oliva, F. (2001). Selección y valuación de carteras. La Habana: Editorial Félix Varela.

Delgado Victore, R. (2010). Dirección integrada de proyectos haciendo uso de las nuevas tecnologías de la informática y las comunicaciones. Santiago de Cuba: Ministerio de Educación Superior.

Delgado, R., López, M. y Galgueras, D. (2011). La evaluación financiera de inversiones: su importancia en las empresas cubanas. Revista Cofin Habana. Revista Cubana de Contabilidad y Finanzas Universidad de La Habana, 3. Recuperado de http://www.cofinhab.uh.cu/index.php/cofin/article/view/30/30

Fernández Carazo, A., Gómez Núñez, T., Guerrero Casas, F. M. y Caballero Fernández, R. (2008). Evaluación y clasificación de las técnicas utilizadas por las organizaciones, en las últimas décadas, para seleccionar proyectos. Revista de Métodos Cuantitativos para la Economía y la Empresa, 5, 67-115. Recuperado de http://www.upo.es/RevMetCuant/art20.pdf

International Organization for Standardization. (2012). Norma ISO 21500:2012. Recuperado de https://www.iso.org/obp/ui\#iso:std:iso:21500:ed-1:v1:es

Ozdemir, S. M., \& Gasimov, R. N. (2004). The analytic hierarchy process and multiobjective 0-1 faculty course assignment. European Journal of Operational Research, 157(2), 398-408. Recuparado de http://www.sciencedirect.com/ science/article/pii/S0377221703001899

Phillips-Wren, G. E., Hahn, E. D., \& Forgionne, G. A. (2004). A multiple criteria framework for evaluation of decision support systems. Omega, 32(4), 323-332. Recuperado de http://www.sciencedirect.com/science/article/pii/ S0305048304000064

Norma ISO, 10 500, 21100

PMBOK. (2013). A guide to the project management body of knowledge (5. ${ }^{\mathrm{a}} \mathrm{ed}$. ) Newtown Square, Pennsylvania, U. S. A.: Project Management Institute, Inc. PMI. (2013). Guía de los fundamentos para la dirección de proyectos (Guía del PMBOK). Pennsylvania, E. U. A.: Project Management Institute, Inc.

Reyes Espinosa, M. (2001). Teoría y práctica de la estructura financiera empresarial y la eficiencia. Estudio de casos en Cuba (Tesis doctoral). Universidad de La Habana, Cuba. 